\title{
642.
}

\section{ON A DIFFERENTIAL RELATION BETWEEN THE SIDES OF A QUADRANGLE.}

[From the Messenger of Mathematics, vol. vi. (1877), pp. 99-101.]

LET the sides and diagonals $Y Z, Z X, X Y, O X, O Y, O Z$ of a quadrangle be $f, g, h, a, b, c$, and let the component triangles be denoted as follows:

$$
\begin{aligned}
& A=\Delta Y Z O,=(b, c, f), \\
& B=\Delta Z X O,=(c, a, g), \\
& C=\Delta X Y O,=(a, b, h), \\
& \Omega=\Delta X Y Z,=(f, g, h),
\end{aligned}
$$

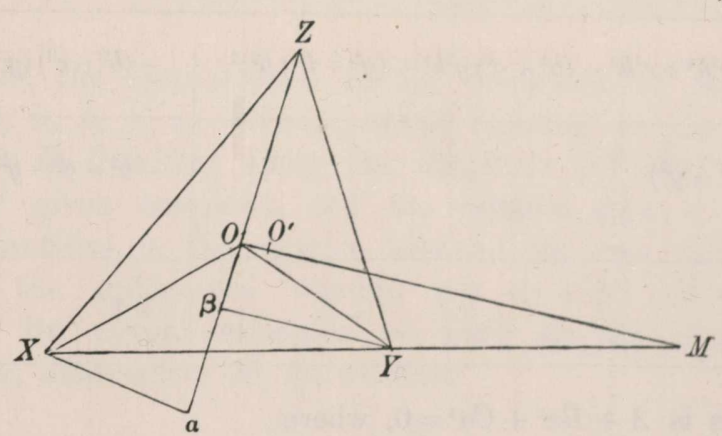

viz. $A, B, C, \Omega$ are the triangles whose sides are $(b, c, f),(c, a, g),(a, b, h),(f, g, h)$ respectively, so that $\Omega=A+B+C$. Then we have between $(a, b, c, f, g, h)$ an equation giving rise to a differential relation, which may be written

$$
\Omega(A a d a+B b d b+C c d c)-(B C f d f+C A g d g+A B h d h)=0 .
$$

This may be proved geometrically and analytically. First, for the geometrical proof, it is enough to prove that, when $a$ and $b$ alone vary, the relation between the increments is $A a d a+B b d b=0$; for then $a$ and $g$ alone varying, the relation between c. $\mathrm{X}$. 
the increments will be $\Omega a d a-C g d g=0$ (as to the negative sign it is clear from the figure that $a, g$ will increase or diminish together): and we thence at once infer the general relation.

We have consequently to prove that, considering $a$ and $b$ as alone variable,

or, what is the same thing,

$$
A a d a+B b d b=0
$$

$$
a d a:-b d b=X O Z: Y O Z \text {. }
$$

The points $X Y Z$ remain fixed; but $O$ moves through the infinitesimal are $O O^{\prime}$, centre $Z$, which may be considered as situate in the right line $O M$ drawn from $O$ at right angles to $Z O$, and meeting $X Y$ produced in the point $M$. And then, writing for a moment $\angle O X Y=X, \angle O Y X=Y, \angle O M Y=M$, we find at once

that is,

$$
\begin{aligned}
d a & =0 O^{\prime} \cos (X+M), \\
-d b & =0 O^{\prime} \cos (Y-M) ;
\end{aligned}
$$

$$
-\frac{d a}{d b}=\frac{\cos (X+M)}{\cos (Y-M)}, \text { or }-\frac{a d a}{b d b}=\frac{a \cos (X+M)}{b \cos (Y-M)} \text {. }
$$

But drawing $X \alpha, Y \beta$ each of them at right angles to $Z O$, we have $a \cos (X+M)=X \alpha$, $b \cos (Y-M)=Y \beta$, and evidently $X O Z: Y O Z=X \alpha: Y \beta$; whence the equation is $-\frac{a d a}{b d b}=\frac{X O Z}{Y O Z}$, which is the required relation.

For the analytical proof, it is to be observed that the relation between $a, b, c$, $f, g, h$ is a quadric relation in the quantities $a^{2}, b^{2}, c^{2}, f^{2}, g^{2}, h^{2}$ respectively; this may be written

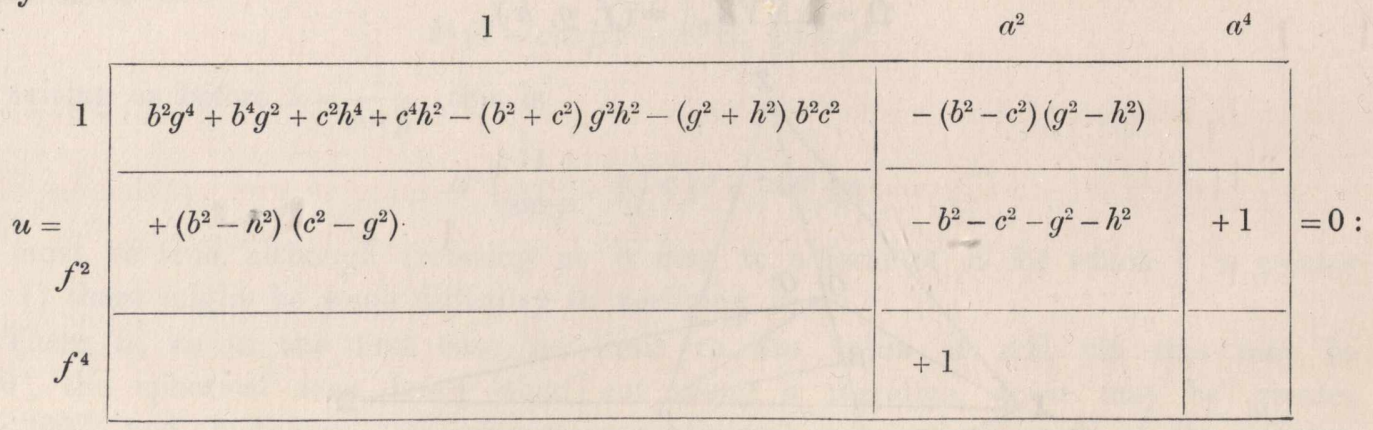

say for a moment this is $A+B a^{2}+C a^{4}=0$, where

$$
\begin{aligned}
& A=b^{2} g^{4}+b^{4} g^{2}+c^{2} h^{4}+c^{4} h^{2} \quad+f^{2}\left(b^{2}-h^{2}\right)\left(c^{2}-g^{2}\right) \\
& -\left(b^{2}+c^{2}\right) g^{2} h^{2}-\left(g^{2}+h^{2}\right) b^{2} c^{2} \\
& B=-\left(b^{2}-c^{2}\right)\left(g^{2}-h^{2}\right) \quad+f^{2}\left(-b^{2}-c^{2}-g^{2}-h^{2}\right)+f^{4} \text {, } \\
& C=\quad f^{2} \text {; }
\end{aligned}
$$

then we have as usual

$$
\frac{d u}{d \cdot a^{2}} a d a+\frac{d u}{d \cdot b^{2}} b d b+\& c .=0
$$


where

But in virtue of $u=0$, we have

$$
\frac{1}{2} \frac{d u}{d \cdot a^{2}}=C a^{2}+\frac{1}{2} B
$$

$$
\left(C a^{2}+\frac{1}{2} B\right)^{2}=C\left(C a^{4}+B a^{2}+A\right)+\frac{1}{4}\left(B^{2}-A C\right),
$$

that is, $\frac{d u}{d \cdot a^{2}}=\sqrt{ }\left(B^{2}-4 A C\right)$; and here $B^{2}-4 A C$ is a quartic function of $f^{2}$, which is easily seen to reduce itself to the form

$$
f^{2}-(g+h)^{2} f^{2}-(g-h)^{2} f^{2}-(b+c)^{2} f^{2}-(b-c)^{2} .
$$

The coefficients of $b d b, c d c$, \&c., are given as expressions of the like form; substituting their values, the differential relation is

$$
\sqrt{ }\left\{f^{2}-(g+h)^{2} f^{2}-(g-h)^{2} f^{2}-(b+c)^{2} f^{2}-(b-c)^{2}\right\} a d a+\& c .=0,
$$

which is, in fact, the foregoing result.

It is right to notice that there are in all 16 linear factors,

$$
\begin{aligned}
& f+g+h, \quad b+c+f, \quad c+a+g, \quad a+b+h \mid \text { say } d, f, g, h, \\
& -f+g+h,-b+c+f,-c+a+g,-a+b+h \quad d^{\prime}, f^{\prime}, g^{\prime}, h^{\prime}, \\
& f-g+h, \quad b-c+f, \quad c-a+g, \quad a-b+h \quad d^{\prime \prime}, f^{\prime \prime}, g^{\prime \prime}, h^{\prime \prime}, \\
& f+g-h, \quad b+c-f, \quad c+a-g, \quad a+b-h \quad d^{\prime \prime \prime}, \quad f^{\prime \prime \prime}, \quad g^{\prime \prime \prime}, \quad h^{\prime \prime \prime} ;
\end{aligned}
$$

and this being so, the coefficients of $a d a, b d b, c d c, f d f, g d g, h d h$, are

respectively.

$$
\begin{aligned}
& \sqrt{ }\left(d d^{\prime} d^{\prime \prime} d^{\prime \prime \prime} \cdot f f^{\prime} f^{\prime \prime} f^{\prime \prime \prime}\right), \quad-\sqrt{ }\left(g g^{\prime} g^{\prime \prime} g^{\prime \prime \prime} \cdot h h^{\prime} h^{\prime \prime} h^{\prime \prime \prime}\right), \\
& \sqrt{ }\left(d d^{\prime} d^{\prime \prime} d^{\prime \prime \prime} \cdot g g^{\prime} g^{\prime \prime} g^{\prime \prime \prime}\right), \quad-\sqrt{ }\left(h h^{\prime} h^{\prime \prime} h^{\prime \prime \prime} \cdot f f^{\prime} f^{\prime \prime} f^{\prime \prime \prime}\right), \\
& \sqrt{ }\left(d d^{\prime} d^{\prime \prime} d^{\prime \prime \prime} \cdot h h^{\prime} h^{\prime \prime} h^{\prime \prime \prime}\right), \quad-\sqrt{ }\left(f f^{\prime} f^{\prime \prime} f^{\prime \prime \prime} \cdot g g^{\prime} g^{\prime \prime} g^{\prime \prime \prime}\right),
\end{aligned}
$$

We may imagine the quadrilateral $Z O X Y$ composed of the four rods $Z O, O X$, $X Y, Y Z$ (lengths $c, a, h, f$ as before) jointed together at the angles, and kept in equilibrium by forces $B, G$ acting along the diagonals $O Y(=b), Z X(=a)$ respectively. We have $c, a, h, f$ given constants, and the relation $\phi(a, b, c, f, g, h)=0$, which connects the six quantities is the relation between the two variable diagonals $(g, b)$; by what precedes, the differential relation $\phi^{\prime} g \cdot d g+\phi^{\prime} b \cdot d b=0$ is equivalent to $\Omega B b d b-C A g d g=0$. By virtual velocities we have as the condition of equilibrium $B d b+G d g=0$; hence, eliminating $d b, d g$ we have

or, say

$$
\frac{B}{\Omega B b}=-\frac{G}{C A g}
$$

$$
\frac{B}{b}:-\frac{G}{g}=\frac{1}{\Delta X Y O \cdot \Delta Z Y O}: \frac{1}{\Delta X Y Z \cdot \triangle Z X O},
$$

viz. the forces, divided by the diagonals along which they act, are proportional to the reciprocals of the products of the two pairs of triangles which stand on these diagonals respectively. The negative sign shows, what is obvious, that the forces must be, one of them a pull, the other a push. 